\title{
Voluntary Nicotine Consumption Triggers In Vivo Potentiation of Cortical Excitatory Drives to Midbrain Dopaminergic Neurons
}

\author{
Stéphanie Caillé, ${ }^{2,3 \star}$ Karine Guillem, ${ }^{4 \star}$ Martine Cador, ${ }^{2,3}$ Olivier Manzoni, ${ }^{1,2}$ and François Georges ${ }^{1,2}$ \\ ${ }^{1}$ Inserm U862, Neurocentre Magendie, Pathophysiology of Synaptic Plasticity Group, ${ }^{2}$ University of Bordeaux, and ${ }^{3}$ Centre National de la Recherche \\ Scientifique (CNRS) UMR 5227, Neuropsychopharmacology of Addiction Group, University of Bordeaux, Bordeaux F-33076, France, and ${ }^{4}$ Center for \\ Neurogenomics and Cognitive Research, VU University Amsterdam, 1081 HV Amsterdam, The Netherlands
}

Active response to either natural or pharmacological reward causes synaptic modifications to excitatory synapses on dopamine (DA) neurons of the ventral tegmental area (VTA). Here, we examine these modifications using nicotine, the main addictive component of tobacco, which is a potent regulator of VTA DA neurons. Using an in vivo electrophysiological technique, we investigated the role of key components of the limbic circuit, the infralimbic cortex (ILCx) and the bed nucleus of the stria terminalis (BNST), in operant behaviors related to nicotine reward. Our results indicated that nicotine self-administration in rats, but not passive delivery, triggers hyperactivity of VTA DA neurons. The data suggest that potentiation of the ILCx-BNST excitatory pathway is involved in these modifications in VTA DA neurons. Thus, recruitment of these specific excitatory inputs to VTA DA neurons may be a neural correlate for the learned association between active responding and the reward experience.

\section{Introduction}

Midbrain dopamine (DA) neurons that originate from the ventral tegmental area (VTA) are important for motivated behaviors toward natural and pharmacological rewards. Supporting this idea, reports in rats indicate that voluntary intake, but not passive administration, of either food or cocaine is equally effective in enhancing synaptic strength onto VTA DA neurons ex vivo (Chen et al., 2008). Thus, the VTA is likely to be a site of convergence for input carrying information on whether the reinforcement is actively obtained or passively received by the animal (Marinelli et al., 2003; Chen et al., 2008).

The bed nucleus of the stria terminalis (BNST) projects to VTA DA neurons and exerts a strong excitatory influence on their firing properties (Georges and Aston-Jones, 2001, 2002). This excitatory projection has been implicated in cocaine and food self-administration (Dumont et al., 2005; Grueter et al., 2006), drug-seeking (Aston-Jones and Harris, 2004), and stressinduced relapse to cocaine (Erb et al., 2001). The efficiency of this BNST-VTA excitatory projection is enhanced in rats performing

Received June 22, 2009; accepted July 18, 2009.

This work was supported by grants from Inserm, Centre National de la Recherche Scientifique, University Bordeaux 2, MILDT/INCa/ Inserm 2006, and Région Aquitaine. We thank Dr. L. Groc for helpful discussions and comments. We thank Ray Cooke and Jane Coulter for their editorial assistance.

*S.C. and K.G. contributed equally to this work.

Correspondence should be addressed to either of the following: François Georges, Inserm U862, Neurocentre Magendie, Pathophysiology of Synaptic Plasticity Group, University of Bordeaux, Bordeaux F-33076, France, E-mail: francois.georges@inserm.fr; or Stéphanie Caillé, CNRS UMR 5227, Neuropsychopharmacology of Addiction Group, University of Bordeaux, Bordeaux F-33076, France, E-mail: stephanie.garnier@u-bordeaux2.fr.

DOI:10.1523/JNEUROSCI.2950-09.2009

Copyright $\odot 2009$ Society for Neuroscience $\quad 0270-6474 / 09 / 2910410-06 \$ 15.00 / 0$ an operant task to obtain food or cocaine. In contrast, no changes were observed in glutamate synaptic transmission in the BNST in rats that received food or cocaine in a passive manner (Dumont et al., 2005). Thus, the difference between active responding versus passive exposure is possibly related to the establishment of reinforcement learning processes, as self-administering animals would be the only ones able to make the action-outcome association. Interestingly, the infralimbic cortex (ILCx) has been involved in the mechanism whereby overtraining comes to produce habitual responses that override goal-directed actions (Coutureau and Killcross, 2003). Moreover, we recently demonstrated that BNST is necessary for the efficient relay of cortical excitation to DA neurons of the VTA (Massi et al., 2008). Are these cortical inputs involved in active responding?

The pharmacological target of nicotine, the main addictive component of tobacco, for the control of VTA DA neuron activity is now well identified (Mameli-Engvall et al., 2006). It is known that acute nicotine acts on midbrain DA neurons to release dopamine in the nucleus accumbens (Pontieri et al., 1996). However, the impact of nicotine reinforcement and learning processes on the activity of the ILCx-BNST glutamatergic afferent pathway to the VTA DA neurons has not been examined. Thus, the present study sought to dissect the network organization of the glutamate-dependent changes underlying learning mechanisms in response to voluntary nicotine consumption. Here, we show that operant nicotine self-administration, but not passive administration, leads to hyperactivity of VTA DA neurons in vivo. In addition, our results suggest that the potentiation of an ILCX-BNST excitatory pathway may be partly responsible for the modifications observed in VTA DA neurons. 


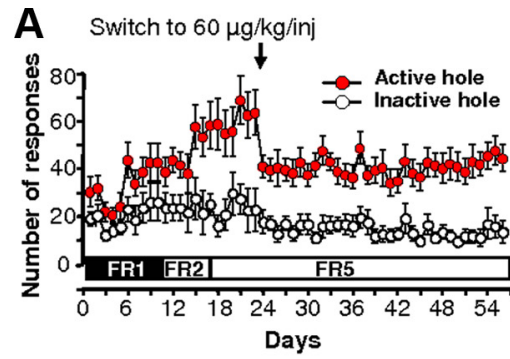

C
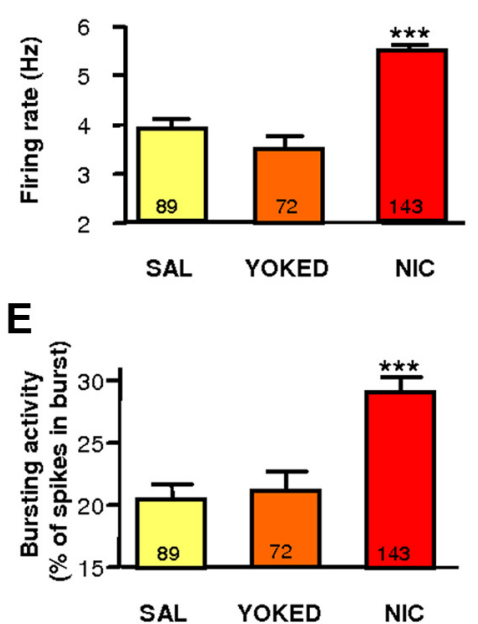

Figure 1. Voluntary nicotine consumption elevates VTA DA neuronal activity. $\boldsymbol{A}$, Nicotine self-administration in the NIC group during a 2 month training under fixed-ratio schedules of reinforcement (FR1, FR2, and FR5). When responding was stable, the nicotine unit dose was increased from 30 to $60 \mu \mathrm{g} / \mathrm{kg} /$ infusion (arrow). Data are shown as mean \pm SEM responses on the active (red circles) and inactive (white circles) nose-pokes. $\boldsymbol{B}, 0$ scilloscope traces of impulses from three VTA DA neurons showing typical recordings of two regular and one fast-firing VTA DA neuron in SAL, YOKED, and NIC rats, respectively. C, Analysis of firing activity of VTA DA neurons in SAL, YOKED, and NIC rats. Bar charts show firing rates as a function of the treatment that each group received. $D$, Histograms showing the distribution of VTA DA firing rates in SAL and NIC rats. Scale on the $y$-axis of the histograms corresponds only to the histogram bars. Solid lines on each of the graphs represent best-fit normal distribution curves for the histogram data. $\boldsymbol{E}$, Analysis of the bursting activity of VTA DA neurons in SAL, YOKED and NIC rats. Bar charts show percentage of spikes in bursts as a function of the treatment that each group received. $\boldsymbol{F}$, The frequency (left axis) or burst firing (right axis) was independent of the amount of nicotine self-administered. The dot chart represents the average firing rate (closed circles) and the average bursting activity (open circles) for all the VTA DA neurons recorded in each rat as a function of the total amount of self-administered nicotine. There is no correlation between bursting or firing activity and the total amount of nicotine self-administered by the NIC rats. Pearson correlation calculation; firing rates: Pearson $r=0.20, R^{2}=0.04$; bursting activity: Pearson $r=0.16, R^{2}=0.03$. A one-sample Kolmogorov-Smirnov test was used to test normal distribution. ${ }^{* * *} p<0.001$ versus VTA DA activity in SAL rats. Numbers refer to numbers of VTA DA neurons recorded.

\section{Materials and Methods}

Animals. Ninety-three male Sprague Dawley rats (Charles River, 175-200 g) were used. They were housed collectively (3/cage) and maintained in rooms at $20-22^{\circ} \mathrm{C}$ with a reversed light/dark cycle (lights off from 9:00 A.M. to 9:00 P.M.). One week before the start of self-administration training, rats were placed on a restricted diet of $20 \mathrm{~g} / \mathrm{d}$ lab chow, sufficient to maintain body weight and growth throughout the experiment. Water was available ad libitum, and food was given after daily testing.

Intravenous nicotine self-administration. Briefly, animals were deeply anesthetized with chloral hydrate $(350 \mathrm{mg} / \mathrm{kg}$, i.p.) and prepared with chronic indwelling SILASTIC jugular catheters as described previously (Guillem et al., 2005). After surgery, animals were flushed daily with 0.2 $\mathrm{ml}$ of an ampicillin solution ( $0.1 \mathrm{~g} / \mathrm{ml}$; PANPHARMA Laboratories) containing heparin $(300 \mathrm{IU} / \mathrm{ml})$ for a minimum of $6 \mathrm{~d}$ of postoperative recovery before the initiation of self-administration training.

Experiments were conducted at the beginning of the dark cycle. Rats were tested in operant chambers $(30 \times 40 \times 37 \mathrm{~cm}$, Imetronic $)$ equipped with two nose-poke devices ("active" and "inactive"). The beginning of the $2 \mathrm{~h}$ self-administration session was indicated by illumination of the house light and a single noncontingent infusion of the drug solution. Activation of the active nose-poke resulted in the infusion of
$100 \mu \mathrm{l}$ of nicotine (NIC, $n=28$, beginning at $30 \mu \mathrm{g} / \mathrm{kg} /$ infusion and increased to $60 \mu \mathrm{g} / \mathrm{kg} /$ infusion) or saline (SAL, $n=27$ ) over $4 \mathrm{~s}$, and was accompanied by the illumination of a white cue-light positioned above the nosepoke hole for 3 s. A 20 s time-out followed each infusion whereby activation of the active nose-poke had no consequences. Inactive nose-poke responses were recorded but had no programmed consequences. In the YOKED group, nicotine infusions were matched to those of the NIC rats, so the YOKED rats never associated discrete stimuli or operant responding with nicotine delivery. Rats were trained for the acquisition of self-administration under a fixedratio (FR) schedule of reinforcement (10 d FR-1; 3 d FR-2; remaining days FR-5). For each rat, training lasted at least 2 months and continued until the in vivo electrophysiological recording.

Osmotic pump implantation. Twenty-four rats were anesthetized with halothane oxygen mixture (1-3\% halothane) and prepared with Alzet osmotic pumps [model 2 ML2 (14 d); Alza Corporation] placed subcutaneously (back of the animal, parallel to the spine). Pumps were filled either with saline (pumpSAL, $n=17$ ) or nicotine tartrate solution (pump-NIC, $n=7$ ). The concentration of nicotine tartrate salt solution was adjusted according to animal weight, resulting in $9 \mathrm{mg} / \mathrm{kg} / \mathrm{d}$ $(3.16 \mathrm{mg} / \mathrm{kg} / \mathrm{d}$, free base) for $6 \mathrm{~d}$. Electrophysiological recordings were performed $24 \mathrm{~h}$ after removal of the pumps.

Stereotaxic surgery. Stereotaxic surgery for electrophysiology experiments was performed under halothane anesthesia as previously described (Georges and Aston-Jones, 2002). Recording pipettes or stimulating electrodes were inserted into the ILCX, the BNST, or the VTA with the skull flat, at the following coordinates: ILCx: $+3.0 \mathrm{~mm}$ from bregma, $0.5 \mathrm{~mm}$ from midline, $4.5 \mathrm{~mm}$ from brain surface; BNST: $-0.3 \mathrm{~mm}$ from bregma; $1.5 \mathrm{~mm}$ from midline, $6.0-7.5 \mathrm{~mm}$ from brain surface; VTA: $-5.3 \mathrm{~mm}$ from bregma; $0.8 \mathrm{~mm}$ from midline, $8.2 \mathrm{~mm}$ from brain surface.

Ventral tegmental area recordings. A glass micropipette (tip diameter $=2-3 \mu \mathrm{m}, 4-6 \mathrm{M} \Omega$ ) filled with a $2 \%$ pontamine sky blue solution in $0.5 \mathrm{~m}$ sodium acetate was lowered into the VTA. DA neurons were identified according to well established electrophysiological features (Grace, 1988; Ungless et al., 2004). These included: (1) an half action potential width $\geq 1.1 \mathrm{~ms}$; (2) slow spontaneous firing rate $(<10 \mathrm{~Hz})$; and (3) single and burst spontaneous firing patterns. Through these electrodes, the extracellular potential was recorded with an Axoclamp2B amplifier in the bridge mode. The extracellular potential amplified 10 times by the Axoclamp2B amplifier was further amplified 100 times and filtered (low-pass filter at $300 \mathrm{~Hz}$ and high-pass filter at 0.5 $\mathrm{kHz}$ ) via a differential AC amplifier (model 1700; A-M Systems). Single neuron spikes were discriminated and digital pulses were collected online using a laboratory interface and software (CED 1401, SPIKE 2; Cambridge Electronic Design).

Bed nucleus stria terminalis recordings. A glass micropipette (tip diameter $=1-2 \mu \mathrm{m}, 10-15 \mathrm{M} \Omega$ ) filled with a $2 \%$ pontamine sky blue solution in $0.5 \mathrm{~m}$ sodium acetate was lowered into the BNST. Through these electrodes, the extracellular potential was recorded as described above for VTA DA neurons. Electrical stimulation of the VTA was used to test for antidromic activation of BNST neurons using high-frequency following and collision methods, as previously described (Georges and Aston- 
Jones, 2002). A bipolar concentric stimulation electrode was inserted into the VTA, and stimulation of the VTA was conducted with a concentric electrode (Phymep). Electrical stimulation (single pulse, $1.0 \mathrm{~mA}$ in intensity, $0.5 \mathrm{~ms}$ in duration) was administered using a square pulse stimulator and stimulus isolator (DS3; Digitimer). Driven impulses were considered antidromic if they met the following criteria: (1) constant latency of spike response, (2) driving by each of the paired stimulus pulses at frequencies of $100 \mathrm{~Hz}$ or greater, and (3) collision of driven spikes by spontaneous impulses occurring within a critical interval approximately equal to the sum of the refractory period plus the driving latency. After isolating a single BNST neuron projecting to the VTA, prestimulation spontaneous activity was recorded to establish baseline activity for at least $5 \mathrm{~min}$. Subsequently, single pulses were delivered to the ILCx every $2 \mathrm{~s}$ at increasing intensity. At least 100 trials were administered per cell for a given intensity.

Electrical stimulation of the infralimbic cortex. Bipolar electrical stimulation of the ILCx was conducted with a concentric electrode $(250 \mu \mathrm{m}$ diameter overall, $100 \mu \mathrm{m}$ diameter inner electrode which extended 100 $\mu \mathrm{m}$ beyond the outer electrode; Phymep). This electrode was inserted into the ILCx. Electrical stimulation (0.1, 0.2, 0.5 and $1.0 \mathrm{~mA}$ for BNST recordings, $0.5 \mathrm{~Hz}, 0.5 \mathrm{~ms}$ duration pulses) was administered using a square pulse stimulator and stimulus isolator (DS3; Digitimer).

Histology. At the end of each recording experiment, the electrode placement was marked with an iontophoretic deposit of pontamine sky blue dye ( $-20 \mu \mathrm{A}$, continuous current for $12-15 \mathrm{~min})$. To mark electrical stimulation sites, $10 \mu \mathrm{A}$ of positive current was passed through the stimulation electrode for $1 \mathrm{~min}$. After the experimental procedures, the animals were deeply anesthetized with halothane (5\%) and decapitated. Brains were removed and snap-frozen in a solution of isopentane at $-70^{\circ} \mathrm{C}$.

Data analysis. Analyses of nicotine self-administration were performed using ANOVA. Data were subjected to a two-way ANOVA, with hole (active-inactive) and training days as within-subject factors. Whenever main factor effects were found, post hoc comparisons were performed using the Newman-Keuls test. $p>0.05$ was considered as not statistically significant (NS).

Two parameters of VTA DA neuron impulse activity were computed over $200 \mathrm{~s}$ epochs after a 5 min stable baseline period: (1) the basal firing rate and (2) the proportion of spikes that occurred in bursts. The onset of a burst was defined as the occurrence of two spikes with an interspike interval $<80 \mathrm{~ms}$ (Grace, 1988). The percentage of spikes in bursts was calculated by dividing the number of spikes occurring in bursts by the total number of spikes occurring in the same period of time.

During electrical stimulation of the ILCx, cumulative peristimulus time histograms (PSTHs, $5 \mathrm{~ms}$ bin width) of BNST activity were generated for each neuron recorded. PSTHs were analyzed to determine excitatory and inhibitory epochs as previously described (Georges and Aston-Jones, 2002). In brief, the mean and SD of counts per bin were determined for a baseline period, defined as the $500 \mathrm{~ms}$ epoch preceding stimulation. The onset of excitation was defined as the first bin whose mean value exceeded mean baseline activity by $2 \mathrm{SD}$, and response offset was determined as the time at which activity had returned to be consistently within $2 \mathrm{SD}$ of baseline. Excitatory response magnitudes $\left(R_{\mathrm{mag}}\right.$ values) were normalized for different levels of baseline impulse activity, allowing comparison of intensity of stimulation on evoked responses independent of effects on baseline activity. $R_{\mathrm{mag}}$ values for excitation were calculated with the following equation: Excitation $R_{\text {mag }}=$ (counts in excitatory epoch $)-($ mean counts per baseline bin $\times$ number of bins in excitatory epoch). Stimulus-induced inhibitions were quantified by a spike2 script written to consider the onset of inhibition as beginning with the first of at least three consecutive bins that fell outside the range of
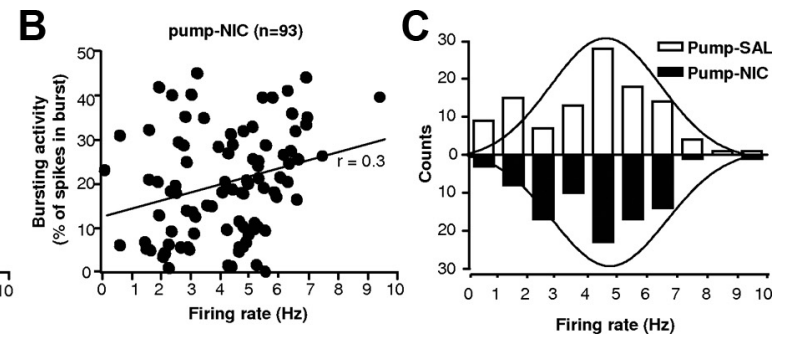

Firing rate $(\mathrm{Hz})$

Figure 2. Analysis of firing activity of VTA DA neurons in rats passively exposed to nicotine. $A, B$, Scatterplots showing the bursting activity as a function of firing rate of individual neurons for rats receiving continuous saline (pump-SAL) or nicotine histogram data. Firing rate and bursting activity of VTA DA neurons for the pump-NIC groups did not differ from pump-SAL.

mean \pm 1 SD of the average firing rate during the baseline control period. Duration of the inhibition was calculated as the difference between the offset and onset of inhibitory period as defined above.

Results are expressed throughout as means \pm SEM. When two means were compared, statistical significance of their difference was assessed using two-tailed paired Student's $t$ tests. For multiple comparisons, values were subjected to a one-way or two-way ANOVA followed by post hoc Dunnett or Newman-Keuls tests.

\section{Results}

\section{History of voluntary nicotine consumption triggers} hyperactivity of dopaminergic neurons

Nicotine self-administration training in $2 \mathrm{~h}$ daily sessions was conducted for at least 2 months. Then, in vivo electrophysiological recordings on NIC ( $n=14$ rats, 143 neurons), YOKED $(n=$ 8 rats, 72 neurons) and SAL ( $n=14$ rats, 89 neurons) rats were performed $24 \mathrm{~h}$ after the last access to the operant chambers. Evolution of the operant responses in the active versus inactive nose-poke modes across the fixed-ratios schedules for the NIC group are illustrated in Figure $1 A$. Rats clearly focused on the active hole and self-administered nicotine, while only rarely visiting the inactive hole (Fig. $1 A$ ) (two-way ANOVA with a Hole $\times$ Training day interaction, $F_{(55,880)}=3.30, p<0.0001$ beginning with trial day 6). Regarding the electrophysiological in vivo measures, VTA DA neuron impulse activity was higher in NIC than in SAL and YOKED (Fig. $1 B-E$ ) (Firing rate: ANOVA; $F_{(2,303)}=$ 37.48, $p<0.0001$; Bursting activity: ANOVA; $F_{(2,303)}=14.74$, $p<0.0001)$. No differences were observed in VTA DA impulse activity between SAL and YOKED groups $(p>0.05)$ (Fig. 1C,E). Typical recording of a fast-firing VTA DA neuron $(>7.5 \mathrm{~Hz})$ of a NIC rat is shown in Figure $1 B$. Firing rates and bursting activity were normally distributed in all groups (Fig. $1 D$, for clarity only VTA DA neuron firing rates of SAL and NIC are shown), which indicates similar neuronal populations (range, 0.5-10 Hz). However, the Gaussian curve was shifted $\sim 1.5 \mathrm{~Hz}$ to the right in NIC compared with SAL (Kolmogorov-Smirnov test, D $=0.1834$, $p<0.01)$. Together, these data show that only the history of voluntary nicotine consumption triggers hyperactivity of VTA DA neurons. Stable patterns of daily nicotine intake $(\sim 450 \mu \mathrm{g} / \mathrm{kg}$ nicotine per day) were rapidly established in rats (data not shown). However, there was no correlation between the change of firing rate or bursting activity and the total amount of nicotine that the rats self-administered (Fig. $1 F$ ). To confirm that modifications in VTA DA neuronal activity were dependent on the 
A

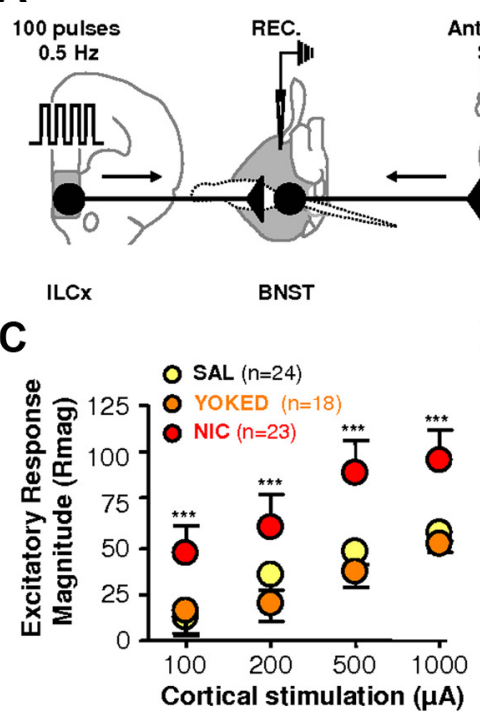

B

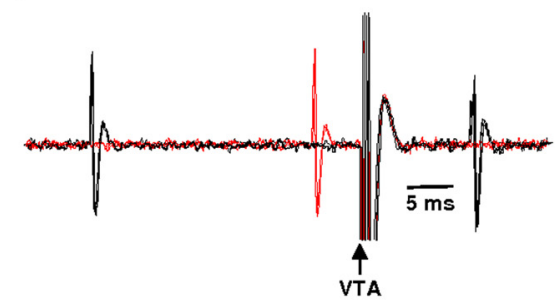

D
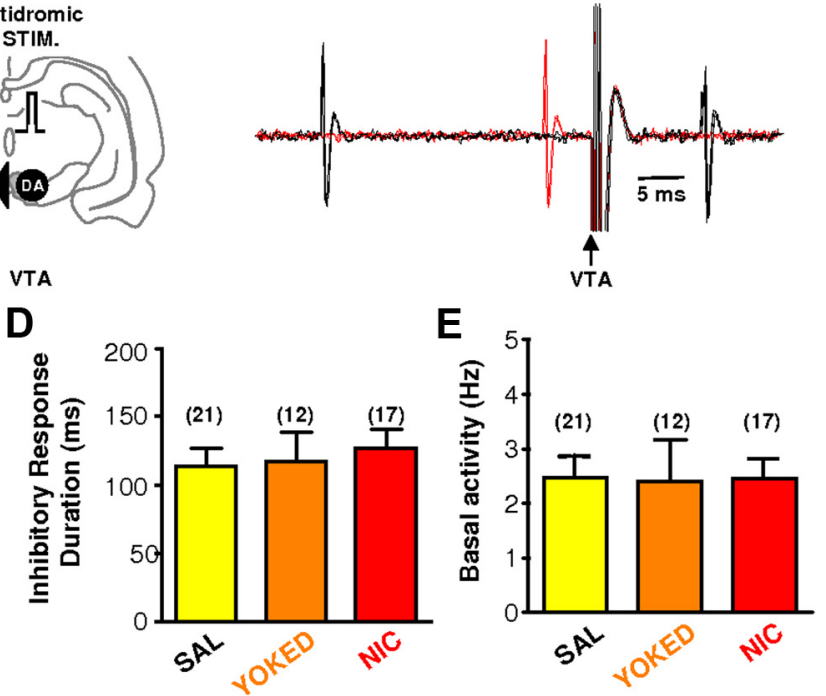

F

F SAL

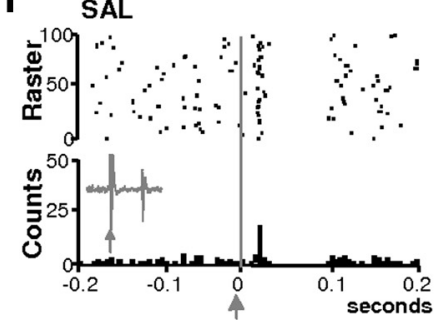

YOKED

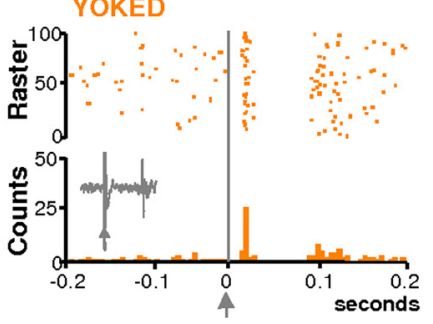

NIC

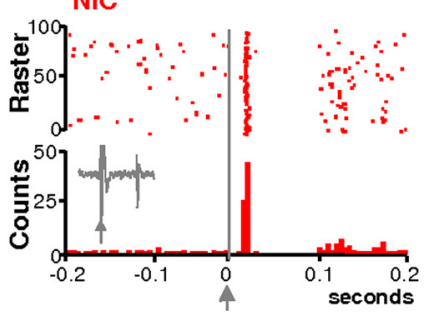

Figure 3. Voluntary nicotine consumption potentiates cortical excitatory drives to the VTA: analysis of input/output tests on BNST neurons projecting to the VTA after stimulation of ILCx in SAL, YOKED, and NIC rats. A, Diagram of the stimulation protocol used in this experiment. Here, the ILCx was stimulated with a 100-pulse train and BNST neurons projecting to the VTA were identified by antidromic activation from the VTA. $\boldsymbol{B}$, Ten consecutive sweeps showing antidromic response of a BNST neuron from VTA stimulation. Stimulation of the VTA $30 \mathrm{~ms}$ after spontaneous spikes elicit driven spikes (black traces). Driven spikes are occluded for similar stimuli delivered $5 \mathrm{~ms}$ after spontaneous impulses indicating collision between spontaneous and driven spikes (red traces). $\boldsymbol{C}$, Increasing intensity of stimulation current in ILCX evoked higher response magnitude of BNST neurons projecting to the VTA. Cortical excitatory strength onto BNST neurons projecting to the VTA was enhanced only in NIC rats. The slope of the relationship between injected current and evoked firing rate is similar in SAL and YOKED animals. Numbers of neurons recorded in each group are mentioned in brackets. ${ }^{* * *} p<0.001$ versus excitatory responses in SAL rats. $\boldsymbol{D}, \boldsymbol{E}$, Graphs illustrating the effects of treatment (SAL, YOKED, and NIC rats) on inhibitory response duration (D) and on basal activity frequency $(\boldsymbol{E})$. Treatment had no effect on inhibition or on basal activity in SAL, YOKED, or NIC rats. $\boldsymbol{F}$, Typical PSTHs illustrate responses of BNST neurons projecting to the VTA on ILCX stimulation $(1000 \mu \mathrm{A})$. In this PSTHs, the stimulus is delivered at time 0 (gray vertical line). Each PSTH consists of 100 trials individually illustrated in the associated raster. Bin width, $5 \mathrm{~ms}$. Numbers in brackets refers to numbers of BNST neurons recorded.

active nicotine intake, we tested another route of drug administration. Therefore, another group of rats were exposed to continuous subcutaneous slow release of nicotine (pump-NIC; $n=7$ rats, 93 neurons, osmotic pumps filled with nicotine at $9 \mathrm{mg} /$ $\mathrm{kg} / \mathrm{d}$, for $6 \mathrm{~d}$ and recordings $24 \mathrm{~h}$ after withdrawal of the pumps). Total intake of nicotine was similar in pump-NIC and NIC groups $(27.1 \pm 2.2 \mathrm{mg}$ and $20.5 \pm 0.4 \mathrm{mg}$, respectively; Student's $t$ test, NS). Control rats underwent similar surgical and recording procedures (pump-SAL; $n=17$ rats, 111 neurons, osmotic pumps filled with $\mathrm{NaCl} 0.9 \%$ ). Recordings were performed $24 \mathrm{~h}$ after the removal of the pumps. The scatterplots in Figure $2 A, B$ report the bursting activity as a function of firing rate for individual neurons. There were small but significant positive correlations between bursting activity (percentage spikes in bursts) and firing rate for VTA DA neurons in pump-SAL $(r=0.3 ; p<0.01$; Pearson's correlation) and in pump-NIC $(r=0.3 ; p<0.01$; Pearson's correlation). Firing rate (Fig. $2 A$ ) (pump-SAL: $4.1 \pm$ $0.2 \mathrm{~Hz}$; pump-NIC: $4.2 \pm 0.2 \mathrm{~Hz}$ ) and bursting activity (Fig. $2 \mathrm{~B}$ ) (pump-SAL: $21.3 \pm 1.4 \%$ of spikes in burst; pump-NIC: $19.6 \pm$ $1.2 \%$ of spikes in burst) of VTA DA neurons for the pump-NIC did not differ from pump-SAL animals and show a similar normal distribution for both groups (Fig. 2C).
History of voluntary nicotine consumption triggers potentiation of cortical and limbic excitatory drives to dopaminergic neurons

In accordance with the idea that glutamatergic inputs to the VTA are crucial for the regulation of DA neuron basal activity, we hypothesized that the hyperactivity of VTA DA neurons was driven by changes in afferent glutamatergic circuitry to the VTA, perhaps from the BNST or the prefrontal cortex (Dumont et al., 2005; Massi et al., 2008; Peters et al., 2008).

To test this hypothesis, we recorded the impulse activity of BNST neurons projecting to the VTA after stimulation of the ILCx (Fig. $3 A, B$ ) after active responding for nicotine (NIC; $n=$ 14 rats, 24 neurons), passive nicotine delivery (YOKED; $n=6$ rats, 18 neurons), or saline exposure (SAL; $n=13$ rats, 24 neurons). Increasing intensity of stimulation current in ILCx evoked a larger response magnitude of BNST neurons projecting to the VTA (Fig. 3C) (two-way ANOVA, $F_{(2,153)}=9.83$, $p<0.001)$. Cortical excitation strength onto VTA-projecting BNST neurons was higher in NIC than in SAL and YOKED (Fig. $3 C, F$ ) (two-way ANOVA, $F_{(3,153)}=5.18 p<0.001$ ). No differences were observed in excitability of VTA-projecting BNST neurons evoked by ILCx stimulation between SAL and 
YOKED groups $(p>0.05)$ (Fig. 3C,F). In addition, cortical evoked inhibition or basal activity of BNST neurons were not significantly affected in SAL, YOKED, or NIC groups $(p>$ 0.05) (Fig. $3 D, E$ ).

\section{Discussion}

The major finding of the present study is that only the combination of chronic nicotine and the learning process associated with the active self-administration procedure leads to a potentiation of VTA DA neuron activity, especially an increase in bursting activity. Accumulating evidence shows: (1) that an increase in glutamatergic drive enhances dopamine neuronal activity and produces the characteristic bursting pattern expressed in vivo (for review, see Marinelli et al., 2006); (2) that NMDA in DA neurons modulate burst firing and DA release in postsynaptic brain regions (Zweifel et al., 2009); and (3) that bursting activity of dopaminergic neurons is sufficient to mediate behavioral conditioning in freely behaving rats (Tsai et al., 2009). In this context, we propose that recruitment of glutamatergic inputs to VTA DA neurons may be a neural correlate for the learned association between active responding and the reward experience. Moreover, our findings are consistent with a recent ex vivo study showing that $24 \mathrm{~h}$ after self-administration of cocaine or natural reward, excitatory strength is increased in VTA DA neurons (Chen et al., 2008). Recently, we established that the BNST efficiently relays ILCx excitation to VTA DA neurons (Massi et al., 2008). Here, we provide evidence that the hyperactivity of DA neurons after voluntary nicotine consumption is driven by changes in afferent excitatory BNST-ILCx circuitry to the VTA. Together, the data reveal the network organization of the glutamate-dependent changes underlying learning mechanisms associated with the active responding developed in rats with a history of voluntary nicotine intake. Nicotine can trigger pharmacological effects as well as associative processes responsible for self-administration maintenance. On the one hand, the pharmacological effects are classically examined in animals sated with the drug. For instance, Rhaman et al. (2004) showed that there was a desensitization phenomenon occurring in nicotinic receptors in both yoked in nicotine self-administrating rats. On the other hand, the associative processes are examined at several time-points in drug-free animals. For instance, at a $24 \mathrm{~h}$ withdrawal time point, all kinds of reinforcement induced a clear hyperactivity of the DA cells as long as animals did active responding to get the reinforcement [for cocaine: Marinelli and White (2000), Chen et al. (2008); for nicotine: present study]. Importantly, only drugs of abuse produced long lasting modifications of the DA cell activity (Chen et al., 2008).

Consistent with our result, it has been found that nicotine self-administration acutely activates the brain reward system in rats (Kenny and Markou, 2006). Moreover, a recent study demonstrated that nicotine self-administration upregulates NMDA subunit expression in the VTA and the central amygdala (CeA) (Kenny et al., 2009). In this context, and since the two key components of the extended amygdala, the BNST and the CeA, receive their main excitatory input from the ILCx (Vertes, 2004), we speculate that: (1) the ILCx exerts a powerful control over the activity of the extended amygdala, and this neuronal circuit is potentiated by a voluntary nicotine consumption; and that (2) the hyperactivity of VTA DA neurons after a voluntary nicotine consumption is NMDA receptor mediated.

In conclusion, the present study emphasizes the fact that active versus passive drug administration leads to different neuroadaptive modifications in the brain. Altogether, our findings suggest that a history of voluntary nicotine intake, but not passive delivery, controls the cortical excitability of BNST projection neurons to the VTA DA neurons. Moreover, the synaptic hyperactivation of VTA DA neurons may be the neural correlate for the reinforcement learning that is expressed only in rats trained for nicotine self-administration. Thus, while DA system phasic firing has a central role in coding positive and negative motivational signals (Schultz, 2007; Matsumoto and Hikosaka, 2009), our findings suggest that changes in the tonic basal activity of DA VTA neurons are important for the signaling of reinforcement learning and memory.

\section{References}

Aston-Jones G, Harris GC (2004) Brain substrates for increased drug seeking during protracted withdrawal. Neuropharmacology 47:167-179.

Chen BT, Bowers MS, Martin M, Hopf FW, Guillory AM, Carelli RM, Chou JK, Bonci A (2008) Cocaine but not natural reward self-administration nor passive cocaine infusion produces persistent LTP in the VTA. Neuron 59:288-297.

Coutureau E, Killcross S (2003) Inactivation of the infralimbic prefrontal cortex reinstates goal-directed responding in overtrained rats. Behav Brain Res 146:167-174.

Dumont EC, Mark GP, Mader S, Williams JT (2005) Self-administration enhances excitatory synaptic transmission in the bed nucleus of the stria terminalis. Nat Neurosci 8:413-414.

Erb S, Salmaso N, Rodaros D, Stewart J (2001) A role for the CRFcontaining pathway from central nucleus of the amygdala to bed nucleus of the stria terminalis in the stress-induced reinstatement of cocaine seeking in rats. Psychopharmacology 158:360-365.

Georges F, Aston-Jones G (2001) Potent regulation of midbrain dopamine neurons by the bed nucleus of the stria terminalis. J Neurosci 21:RC160.

Georges F, Aston-Jones G (2002) Activation of ventral tegmental area cells by the bed nucleus of the stria terminalis: a novel excitatory amino acid input to midbrain dopamine neurons. J Neurosci 22:51735187.

Grace AA (1988) In vivo and in vitro intracellular recordings from rat midbrain dopamine neurons. Ann N Y Acad Sci 537:51-76.

Grueter BA, Gosnell HB, Olsen CM, Schramm-Sapyta NL, Nekrasova T, Landreth GE, Winder DG (2006) Extracellular-signal regulated kinase 1-dependent metabotropic glutamate receptor 5-induced long-term depression in the bed nucleus of the stria terminalis is disrupted by cocaine administration. J Neurosci 26:3210-3219.

Guillem K, Vouillac C, Azar MR, Parsons LH, Koob GF, Cador M, Stinus L (2005) Monoamine oxidase inhibition dramatically increases the motivation to self-administer nicotine in rats. J Neurosci 25:85938600.

Kenny PJ, Markou A (2006) Nicotine self-administration acutely activates brain reward systems and induces a long-lasting increase in reward sensitivity. Neuropsychopharmacology 31:1203-1211.

Kenny PJ, Chartoff E, Roberto M, Carlezon WA Jr, Markou A (2009) NMDA receptors regulate nicotine-enhanced brain reward function and intravenous nicotine self-administration: role of the ventral tegmental area and central nucleus of the amygdala. Neuropsychopharmacology $34: 266-281$.

Mameli-Engvall M, Evrard A, Pons S, Maskos U, Svensson TH, Changeux JP, Faure P (2006) Hierarchical control of dopamine neuron-firing patterns by nicotinic receptors. Neuron 50:911-921.

Marinelli M, White FJ (2000) Enhanced vulnerability to cocaine selfadministration is associated with elevated impulse activity of midbrain dopamine neurons. J Neurosci 20:8876-8885.

Marinelli M, Cooper DC, Baker LK, White FJ (2003) Impulse activity of midbrain dopamine neurons modulates drug-seeking behavior. Psychopharmacology 168:84-98.

Marinelli M, Rudick CN, Hu XT, White FJ (2006) Excitability of dopamine neurons: modulation and physiological consequences. CNS Neurol Disord Drug Targets 5:79-97.

Massi L, Elezgarai I, Puente N, Reguero L, Grandes P, Manzoni OJ, Georges F (2008) Cannabinoid receptors in the bed nucleus of the stria terminalis control cortical excitation of midbrain dopamine cells in vivo. J Neurosci 28:10496-10508. 
Matsumoto M, Hikosaka O (2009) Two types of dopamine neuron distinctly convey positive and negative motivational signals. Nature 459 : $837-841$

Peters J, LaLumiere RT, Kalivas PW (2008) Infralimbic prefrontal cortex is responsible for inhibiting cocaine seeking in extinguished rats. J Neurosci 28:6046-6053.

Pontieri FE, Tanda G, Orzi F, Di Chiara G (1996) Effects of nicotine on the nucleus accumbens and similarity to those of addictive drugs. Nature 382:255-257.

Rahman S, Zhang J, Engleman EA, Corrigall WA (2004) Neuroadaptive changes in the mesoaccumbens dopamine system after chronic nicotine self-administration: a microdialysis study. Neuroscience 129:415-424.

Schultz W (2007) Behavioral dopamine signals. Trends Neurosci 30:203-210
Tsai HC, Zhang F, Adamantidis A, Stuber GD, Bonci A, de Lecea L, Deisseroth $\mathrm{K}$ (2009) Phasic firing in dopaminergic neurons is sufficient for behavioral conditioning. Science 324:1080-1084.

Ungless MA, Magill PJ, Bolam JP (2004) Uniform inhibition of dopamine neurons in the ventral tegmental area by aversive stimuli. Science 303:2040-2042.

Vertes RP (2004) Differential projections of the infralimbic and prelimbic cortex in the rat. Synapse 51:32-58.

Zweifel LS, Parker JG, Lobb CJ, Rainwater A, Wall VZ, Fadok JP, Darvas M, Kim MJ, Mizumori SJ, Paladini CA, Phillips PE, Palmiter RD (2009) Disruption of NMDAR-dependent burst firing by dopamine neurons provides selective assessment of phasic dopamine-dependent behavior. Proc Natl Acad Sci U S A 106:7281-7288. 